\title{
Association between dietary patterns and handgrip strength: analysis of the Korean National Health and Nutrition Examination Survey data between 2014 and 2017
}

\author{
Yunkoo Kang \\ Jieun Kim \\ Kyung Hee University \\ Do-Yeon Kim \\ Kyung Hee University \\ Seung Kim \\ Yonsei University College of Medicine \\ Sowon Park \\ Yonsei University College of Medicine \\ Hyunjung Lim \\ Kyung Hee University \\ Hong Koh ( $\nabla$ khong@yuhs.ac)
}

Yonsei University Wonju College of Medicine https://orcid.org/0000-0003-1712-2138

\section{Research}

Keywords: diet, handgrip strength, nutrition, dietary pattern, children and adolescents, KNHANES

Posted Date: April 15th, 2020

DOl: https://doi.org/10.21203/rs.3.rs-21964/v1

License: (c) (1) This work is licensed under a Creative Commons Attribution 4.0 International License. Read Full License

Version of Record: A version of this preprint was published at Nutrients on October 5th, 2020. See the published version at https://doi.org/10.3390/nu12103048. 


\section{Abstract}

Background Non-invasive anthropometric measurement methods such as those for measuring height and weight are crucial in pediatric patients. However, research focusing on the association between the type of dietary pattern and handgrip strength and handgrip-to-weight ratio in adolescents has not been carried out yet.

Methods The current work aims to investigate the association between handgrip strength and dietary patterns in Korean adolescents. This cross-sectional analyses of the 2014-2017 Korean National Health and Nutrition Examination Survey assessed 2,327 adolescent (aged 10-18 years) who had measured handgrip strength and analyzed association between dietary pattern. The mean of three handgrip strength measurements, per hand, was used in the analysis. Handgrip-toweight ratio was calculated by the following equation: Handgrip strength/body weight $(\mathrm{kg}) \times 100)$. The overall food intake data were subcategorized into 25 food groups from the Korean Nutrient Database. The clusters were examined for nutritional values, and the ready-to-eat, balanced, and western-style fast-food clusters were ultimately generated. The association between handgrip strength, handgrip-to-weight ratio, and dietary intake was analyzed.

Results Overall, $85.6 \%$ of the participants were assigned to a ready-to-eat dietary pattern, $9.3 \%$ to a western-style fastfood dietary pattern, and $5.1 \%$ to a balanced dietary pattern. Compared with the participants following a balanced dietary pattern, those following a ready-to-eat dietary pattern were shown to have significantly lower handgrip strength and handgrip-to-weight ratio. However, no significant association was found between handgrip strength or handgrip-toweight ratio when the western-style fast-food dietary pattern was compared to the balanced dietary pattern.

Conclusions Decreased handgrip strength and handgrip-to-weight ratio value in participants following ready-to-eat dietary patterns indicate a diffuse problem in adolescents' health and possibly imply an association between reduced muscle quality and dietary pattern. Moreover, the overall environmental factors potentially inducing such unhealthy dietary preferences should be investigated, and appropriate lifestyle changes in Korean adolescents should be encouraged.

\section{Background}

Non-invasive anthropometric measurements methods such as those for measuring height and weight are crucial for pediatric patients.(1-3) In fact, anthropometric measurements can help the decision-making process about the need for additional tests. In this context, muscle strength has recently been recognized as an essential measurement.(4-6) Although muscle strength is a crucial factor for muscular force exertion, several other factors are known to be involved. An individual with high muscle strength is likely to follow an appropriate diet as well as a regular physical activity schedule, in addition to being in a healthy psychological state. In fact, several studies have reported an association between sarcopenia and obesity, metabolic syndrome, non-alcoholic fatty liver disease (NAFLD), and even mental disorders.(7-9) Skeletal-muscle mass and quality can be assessed by either dual-energy X-ray absorptiometry or bioelectrical impedance analysis.(10) However, these methods are impractical to perform in clinical settings, especially in the case of children and adolescents. Nevertheless, measuring handgrip strength (HGS) is a simple and inexpensive way to evaluate muscle strength.(11-13) Furthermore, muscle quality can be estimated when handgrip strength is divided by body weight. Moreover, HGS has shown to be associated with many diseases in children, such as metabolic syndrome and NAFLD. $(11,14)$

Adequate dietary intake is essential for children and adolescents' health. Unbalanced dietary-intake habits are known to contribute to obesity in adolescents $(15,16)$; besides, they are associated with major health problems, including metabolic syndrome, mental disorders, and even cancer.(17) Moreover, chronic conditions such as adolescent obesity 
are likely to continue and even worsen in young adults.(18) According to several studies, balanced dietary intake is essential for both adolescents and adults since it can affect the whole life span.

Infectious diseases, mental disorders, alcohol and/or drug abuse, nutrition deficiencies, obesity, and physical inactivity are the primary health risks for adolescents.(19) Moreover, nutritional inadequacies (e.g., undernutrition, overnutrition, picky eating) are known for being common causes of several pathologies in adolescents.(20) On the other hand, many studies have demonstrated the decrease in handgrip strength in the pediatric population. $(5,8,11-13)$ However, the association of the dietary intake pattern with handgrip strength and handgrip-strength-to-weight ratio in adolescents has not been thoroughly investigated yet. In this study, we investigated the association between handgrip strength and dietary intake patterns in Korean adolescents.

\section{Methods}

\section{Study population}

We collected data from the 2014-2017 Korean National Health and Nutrition Examination Survey (KNHANES), a nationally representative cross-sectional survey carried out by the Korea Center for Disease Control and Prevention (KCDC) every 3-4 years. The scope of KNHANES is to assess the health status of the Korean population by using a multistage, clustered, stratified, and rolling sampling method.(21) This study was conducted in accordance with the guidelines of the Declaration of Helsinki (1964), and its protocol was approved by the Institutional Review Board (IRB) of the KCDC. Informed consent was obtained from all participants at the time of the survey. The Kyung Hee Institutional Review Board approved this study protocol (KHSIRB-20-052(EA)).

We initially collected data on a total of 2,988 subjects from the 2014-2017 KNHANES database; thereafter, we excluded non-adolescent subjects (i.e., $\geq 19$ years or $<10$ years old) $(n=340)$ as well as subjects with extreme energy-intake levels (i.e., $<500 \mathrm{kcal} /$ day or $>5,000 \mathrm{kcal} /$ day $)(n=41)$ and subjects without height, weight, and/or HGS data $(n=280)$. In total, 2,327 participants were eventually included in our study (Fig. 1).

\section{Assessment of socioeconomic data and physical activity}

Sociodemographic data of the study participants included age, house income level, province, and physical activity level. Physical activity was defined using the metabolic equivalent task (MET) score according to the scoring protocol of the Korean Physical Activity Questionnaire short form.(22) Physical activity was categorized according to the total MET score as inactive (<600 MET-min/week), active (600-3,000 MET-min/week), or health-enhancing (>3,000 METmin/week).

\section{Anthropometric measurements}

Height (Seca 225; SECA, Hamburg, Germany), weight (GL-6000-20; CASKOREA Co., Ltd., Seoul, Korea), and waist circumference (Seca 200; SECA, Hamburg, Germany) were measured by trained medical staff using standardized techniques and calibrated equipment. Body mass index (BMI) was thereby calculated as weight (kg) divided by height squared $\left(\mathrm{m}^{2}\right)$. The 2017 Korean Children and Adolescents Growth Standard values were used for anthropometric assessment and BMI age- and sex-specific reference data.(23) Blood pressure (BP) was measured before and after a 5minute interval, and the mean value of the two measurements was implemented in our analysis.

\section{Measurement of handgrip strength}

HGS was measured thrice for each hand using a digital grip strength dynamometer (TKK 5401; Takei Scientific Instruments Co., Ltd., Tokyo, Japan). All participants were instructed to hold the dynamometer in an upright standing 
position with the arms by their sides and to squeeze the grip with full force for 3 seconds each time. The mean of three trials was used for data analysis. Handgrip-strength-to-weight ratio (HGSWR) was determined by the following equation: HGS/body weight $(\mathrm{kg}) \times 100 .(11-13)$

\section{Dietary intake assessment and food grouping}

Dietary intake was assessed using the single 24-hour diet recall method through home interviews by trained interviewers, 1 week after the health interview and examination. Nutrient intake was calculated by using the Korean Foods and Nutrients Database of the Rural Development Administration.(24-26)

Food data were categorized into 25 food groups by using Korean Nutrient Database. Food types were divided into 18 groups (grains, potatoes, sugars, beans, nuts, vegetables, mushrooms, fruits, meats, eggs, fish, seaweeds, dairy products, oils, beverages, seasonings, processed foods and etc.) in the Korean Nutrient Database to simplify the interpretation of components. Due to the high intake rate of grains and their derivatives in Korea, we further divided this food group into four subgroups based on their nutritional profiles: white rice, other grains, wheat and bread, and noodles. Besides, kimchi - a traditional fermented-cabbage-based food - was also separated from the vegetable group since it is a commonly consumed side dish in Korea. Ultimately, the beverages group was further divided into soda, sugar-sweetened beverages (SSBs), and non-sugar beverages based on sugar content; in addition, alcohol was removed from the group due to the age of the participants.

\section{Dietary pattern analysis}

Dietary patterns were generated by a k-means cluster analysis using SAS FASTCLUS (SAS Institute Inc.) based on the gram per day ( $\mathrm{g} /$ day) percentage of total energy contribution for each food group. This k-means procedure generates clusters by comparing the Euclidean distance between each subject. Two to five solutions were examined to assess which cluster set was more suitable for the determination of the dietary patterns. The content of the clusters was examined for its nutritional value, and a three-cluster set was eventually selected. Three types of dietary patterns were thereby identified and named according to the food groups with high consumption: the ready-to-eat dietary pattern, which contained only white rice, eggs, fish, dairy products, and processed food. The western-style fast-food dietary pattern, which was based on wheat/bread, noodles, sugars, meat, oils, sodas (sweetened and unsweetened), and seasonings. The balanced dietary pattern, which was based on whole grains, potatoes, beans, nuts, vegetables, mushrooms, fruits, eggs, fish, seaweed, and dairy products.

\section{Statistical analysis}

Statistical analysis was performed using PROC SURVEY in SAS (SAS Institute, Cary, NC, USA) to take into account the complex sampling design and appropriate sampling weights for the national survey. The k-means procedure was used as an algorithm in the cluster analysis which is commonly used to classify individuals directly extraction of patterns on 25 food groups categories dataset.

The chi-squared test was used to compare categorical variables such as age, sex, household income, province, and physical activity. The descriptive comparisons were presented as least squares means \pm standard error (SE), adjusted for age, sex, BMI, and energy intake ( $\mathrm{kcal})$, MET score (METs), and house income using ANCOVA. Finally, multiple regression analysis was used to evaluate HGS as a function of the food components.

All analyses were performed using SAS version 9.4 (SAS Institute, Cary, NC, USA). Statistical significance was defined as a $\mathrm{p}$-value $<0.05$.

\section{Results}


Most of the participants were categorized into the ready-to-eat group. About $85.6 \%$ of the subjects were attributed to a ready-to-eat dietary pattern, $9.3 \%$ of the subjects to a western-style fast-food dietary pattern, and $5.1 \%$ participants to a balanced dietary pattern.

Table 1 shows the general characteristics of the study participants as separated by dietary patterns. In the western-style fast-food group, $51.1 \%$ of the participants were $16-18$ years old, while $18.4 \%$ were $10-12$ years old $(p<0.001)$. The western-style fast-food group had the highest proportion of boys $(68.6 \%, p<0.001)$. With regards to anthropometric variables, adjusted BMI $(20.8 \pm 0.3 p<0.001)$, BP (systolic: $110.8 \pm 0.8$, diastolic: $68.0 \pm 0.7, p<0.001)$, and adjusted BP (systolic: $109.0 \pm 0.8$, diastolic: $66.6 \pm 0.7, \mathrm{p}<0.001$ ) were the highest in the western-style fast-food group. HGS and HGSWR were higher in participants with a balanced dietary pattern than in participants with a ready-to-eat pattern group following age-, sex-, and total energy intake-based adjustments $(26.6 \pm 0.7$ and $50.0 \pm 1.0, p<0.001)$. 
Table 1

General characteristics of 10-18-year-old children and adolescents according to the three dietary patterns ${ }^{1}$.

balanced

$(n=118 ; 5.1 \%)$ ready-to-eat

$(n=1,992 ; 85.6 \%)$ western-style fast-food

p-value

$(n=217 ; 9.3 \%)$

Sociodemographics

Age $<0.001$ *

$10-12$ years

30.6

$(4.4)^{2}$

31.6

(1.2)

18.4

(2.8)

13-15 years $\quad 36.5$

(4.7)

32.6

(1.4)

30.5

16-18 years

32.8

(5.0)

(1.3)

51.1

(3.8)

Sex

$\begin{array}{ll}\text { Boys } & 51.9 \\ \text { Girls } & 48.9\end{array}$

(4.9)

(1.3)

68.6

(1.3)

31.4

0.099

Household income

Low (Q1)

Low-middle

(Q2)

49.4

(1.3)

$<0.001^{*}$

$\begin{array}{llllll}\text { Middle-high } & 22.6 & (4.3) & 34.2 & 34.2\end{array}$

High (Q4)

34.7

$(5.2)$

31.1

(1.6)

31.1

(1.7)

Region

11.5

(2.4)

11.5

(2.4)

23.3

(1.3)

29.4

Urban

62.8

(5.1)

60.1

(1.7)

(1.7)

39.9

Physical activity

Inactive

Active

4.7

(0.5)

(1.2)
85.8

83.0
(0.9)

(2.2)
0.216
9.5

11.8
0.193

$\begin{array}{ll}53.5 & (4.0) \\ 46.5 & (4.0)\end{array}$

1. Dietary patterns were determined by cluster analysis using dietary intake (g/day) consumed.

2. Values are presented as \% (SE). Data were weighted to represent children and adolescents aged 10-18 years from KNHANES 2014-2017.

3. Values are presented as Mean \pm SE. $95 \% \mathrm{Cl}$.

*P-values for differences using the chi-squared test for proportions and analysis of variance for means.

${ }^{\dagger}$ P-values for differences using ANCOVA and adjusting for age, sex, and total energy intake (kcal).

${ }^{+\dagger}$ Different superscript letters represent the results of the post hoc tests. Adjustment for multiple comparisons: Bonferroni.

BMI, body mass index; HGS, handgrip strength; HGSWR, handgrip-strength-to-weight ratio; BP, blood pressure; SBP, systolic blood pressure; DBP, diastolic blood pressure. 


\begin{tabular}{|c|c|c|c|c|c|c|}
\hline \multirow[b]{3}{*}{$\begin{array}{l}\text { Health } \\
\text { enhancing }\end{array}$} & \multicolumn{2}{|c|}{ balanced } & ready-to-eat & \multicolumn{2}{|c|}{ western-style fast-food } & \multirow[t]{2}{*}{$p$-value } \\
\hline & \multicolumn{2}{|c|}{$(n=118 ; 5.1 \%)$} & $(n=1,992 ; 85.6 \%)$ & \multicolumn{2}{|l|}{$(n=217 ; 9.3 \%)$} & \\
\hline & 5.5 & (1.2) & 78.2 & 16.4 & & \\
\hline \multicolumn{7}{|c|}{ Anthropometrics } \\
\hline BMI & \multicolumn{2}{|c|}{$20.6 \pm 0.4(19.8-21.4)^{3}$} & $20.8 \pm 0.1(20.6-21.0)$ & \multicolumn{2}{|c|}{$20.6 \pm 0.4(20.9-22.1)$} & 0.06 \\
\hline BMI adjusted & \multicolumn{2}{|c|}{$20.4 \pm 0.4(19.7-21.2)$} & $20.6 \pm 0.1(20.4-20.8)$ & \multicolumn{2}{|c|}{$20.8 \pm 0.3(20.3-21.4)$} & $<.001^{t+}$ \\
\hline $\begin{array}{l}\text { BMl< }<5 \text { th } \\
\% \text { tile }\end{array}$ & 86.6 & (3.9) & $(0.8)$ & (2.7) & & 0.40 \\
\hline $\begin{array}{l}\text { 95th \%tile } \leq \\
\text { BMl }\end{array}$ & 13.4 & $(3.9)$ & 14.2 & 17.7 & & \\
\hline \multicolumn{7}{|l|}{ HGS } \\
\hline HGS & \multicolumn{2}{|c|}{$27.6 \pm 1.1(25.4-29.7)$} & $26.1 \pm 0.3(25.5-26.6)$ & \multicolumn{2}{|c|}{$30.1 \pm 0.8(29.1-32.1)$} & $<0.001^{\dagger}$ \\
\hline HGS adjusted & \multicolumn{2}{|c|}{$26.6 \pm 0.7(25.3-27.9)$} & $25.3 \pm 0.2(25.0-25.7)$ & \multicolumn{2}{|c|}{$25.8 \pm 0.4(24.9-26.6)$} & $<.001^{+\dagger}$ \\
\hline HGSWR & \multicolumn{2}{|c|}{$50.7 \pm 1.3(48.2-53.3)$} & $47.8 \pm 0.3(47.1-48.4)$ & \multicolumn{2}{|c|}{$51.8 \pm 1.0(49.8-53.8)$} & $<0.001^{\dagger}$ \\
\hline $\begin{array}{l}\text { HGSWR } \\
\text { adjusted }\end{array}$ & \multicolumn{2}{|c|}{$50.0 \pm 1.0(47.9-52.0)^{\mathrm{a}}$} & $47.3 \pm 0.3(46.8-47.9)^{b}$ & \multicolumn{2}{|c|}{$47.7 \pm 0.8(46.2-49.2)^{a b}$} & $<.001^{t+}$ \\
\hline \multicolumn{7}{|l|}{ BP } \\
\hline SBP & \multicolumn{2}{|c|}{$\begin{array}{l}108.0 \pm 1.0(105.9- \\
109.7)\end{array}$} & $108.1 \pm 0.3(107.5-108.6)$ & \multicolumn{2}{|c|}{$110.8 \pm 0.8(109.3-112.3)$} & $<0.001^{\dagger}$ \\
\hline SBP adjusted & $\begin{array}{l}107.8 \\
\pm\end{array}$ & $\begin{array}{l}1.0(106.2- \\
109.9)\end{array}$ & $\begin{array}{l}107.9 \pm 0.3(107.4- \\
108.5)\end{array}$ & $\begin{array}{l}109.0 \pm 0.8(107.6- \\
110.5)\end{array}$ & $<0.0$ & $1^{+t}$ \\
\hline DBP & $66.2 \pm$ & $1.0(64.3-68.1)$ & $66.2 \pm 0.2(65.7-66.6)$ & $68.0 \pm 0.7(66.0-69.3)$ & $<0.0$ & \\
\hline DBP adjusted & $65.9 \pm$ & $.9(64.1-67.7)$ & $65.6 \pm 0.2(65.2-66.1)$ & $66.6 \pm 0.7(65.2-67.9)$ & $<0.0$ & $1^{+t}$ \\
\hline \multicolumn{7}{|c|}{ 1. Dietary patterns were determined by cluster analysis using dietary intake ( $\mathrm{g} /$ day) consumed. } \\
\hline \multicolumn{7}{|c|}{$\begin{array}{l}\text { 2. Values are presented as \% (SE). Data were weighted to represent children and adolescents aged } 10-18 \text { years from } \\
\text { KNHANES } 2014-2017 \text {. }\end{array}$} \\
\hline 3. Values are $\mathrm{pr}$ & ented as & Mean \pm SE. $95 \% \mathrm{Cl}$ & & & & \\
\hline *P-values for di & erences & sing the chi-square & test for proportions and ar & alysis of variance for me & ans. & \\
\hline${ }^{\dagger}$ P-values for $d$ & erences & Ising ANCOVA and & adjusting for age, sex, and $t$ & otal energy intake (kcal). & & \\
\hline $\begin{array}{l}\text { †† Different sup } \\
\text { Bonferroni. }\end{array}$ & script let & ers represent the re & sults of the post hoc tests. & Adjustment for multiple cc & mparis & ons: \\
\hline
\end{tabular}


The food and nutrient intake rates of the study participants are summarized in Table 2. Significant differences in total energy (kcal/day), protein ( $\mathrm{g} /$ day), fat (g/day), and carbohydrate (g/day) intake according to the three dietary patterns were detected $(p<0.05)$. 
Table 2

Nutrients and food intake of 10-18-year-old children and adolescents according to the three dietary patterns.

\begin{tabular}{|c|c|c|c|c|c|c|c|c|c|c|}
\hline \multirow{3}{*}{ Nutrients } & \multirow{2}{*}{\multicolumn{3}{|c|}{$\begin{array}{l}\text { balanced } \\
(n=118 ; 5.1 \%)\end{array}$}} & \multirow{2}{*}{\multicolumn{3}{|c|}{$\begin{array}{l}\text { ready-to-eat } \\
(\mathrm{n}=1,992 ; 85.6 \%)\end{array}$}} & \multicolumn{4}{|c|}{ western-style fast-food } \\
\hline & & & & & & & \multicolumn{3}{|c|}{$(n=217 ; 9.3 \%)$} & \multirow[t]{2}{*}{$\mathrm{p}$-value } \\
\hline & & & & & & & & & & \\
\hline Total energy (kcal/day) & 2418.5 & \pm 83.4 & a & 2054.9 & \pm 19.6 & $\mathrm{~b}$ & 2625.2 & \pm 72.6 & a & $<0.05^{\dagger}$ \\
\hline Protein (g/day) & 67.5 & \pm 2.4 & $\mathrm{~b}$ & 76.7 & \pm 0.6 & a & 80.3 & \pm 2.8 & a & $<0.001^{t+}$ \\
\hline Fat (g/day) & 45.8 & \pm 2.2 & c & 59.7 & \pm 0.5 & $\mathrm{~b}$ & 64.3 & \pm 1.8 & $\mathrm{a}$ & $<0.05^{t+}$ \\
\hline Carbohydrate (g/day) & 367.9 & \pm 7.4 & a & 314.9 & \pm 1.6 & $\mathrm{~b}$ & 300.4 & \pm 5.5 & c & $<0.001^{t+}$ \\
\hline \multicolumn{11}{|l|}{ Food groups(g/day) } \\
\hline White rice & 137.8 & \pm 9.7 & $\mathrm{~b}$ & 165.0 & \pm 2.8 & a & 106.5 & \pm 7.3 & c & $<0.05^{+\dagger}$ \\
\hline Wholegrain & 35.3 & \pm 5.0 & a & 26.7 & \pm 1.3 & $\mathrm{ab}$ & 19.7 & \pm 3.7 & $\mathrm{~b}$ & $<0.05^{+\dagger}$ \\
\hline Wheat/ bread & 66.5 & \pm 12.5 & $\mathrm{~b}$ & 92.9 & \pm 2.8 & a & 104.7 & \pm 8.5 & a & $<0.05^{t+}$ \\
\hline Noodle & 47.5 & \pm 9.8 & & 58.1 & \pm 2.4 & & 41.2 & \pm 7.1 & & \\
\hline Potatoes & 44.8 & \pm 14.4 & & 28.7 & \pm 1.6 & & 30.4 & \pm 4.9 & & \\
\hline Sugars & 12.1 & \pm 2.2 & & 15.4 & \pm 0.8 & & 14.8 & \pm 2.1 & & \\
\hline Beans & 23.1 & \pm 6.0 & & 19.8 & \pm 1.3 & & 16.6 & \pm 3.8 & & \\
\hline Nuts & 5.8 & \pm 2.5 & & 2.5 & \pm 0.3 & & 2.2 & \pm 0.5 & & \\
\hline Vegetables & 138.2 & \pm 20.5 & a & 132.7 & \pm 3.5 & $\mathrm{ab}$ & 99.1 & \pm 8.9 & $\mathrm{~b}$ & $<0.01^{t+}$ \\
\hline Kimchi & 56.4 & \pm 6.5 & & 62.2 & \pm 2.0 & & 56.8 & \pm 7.9 & & \\
\hline Mushrooms & 8.8 & \pm 3.8 & & 5.4 & \pm 0.5 & & 4.1 & \pm 0.9 & & \\
\hline Fruits & 865.5 & \pm 35.5 & a & 103.2 & \pm 3.6 & $\mathrm{~b}$ & 66.4 & \pm 11.2 & $\mathrm{c}$ & $<0.01^{+\dagger}$ \\
\hline Meat and its products & 77.9 & \pm 11.4 & c & 134.7 & \pm 3.2 & $\mathrm{~b}$ & 199.2 & \pm 13.8 & a & $<0.001^{t+}$ \\
\hline Eggs & 31.5 & \pm 4.9 & $a b$ & 32.4 & \pm 1.2 & a & 20.2 & \pm 2.7 & $b$ & $<0.001^{t+}$ \\
\hline Fish & 33.7 & \pm 5.2 & $a b$ & 38.6 & \pm 1.9 & a & 23.0 & \pm 5.1 & $\mathrm{~b}$ & $<0.05^{+\dagger}$ \\
\hline Seaweed & 3.2 & \pm 0.9 & a & 2.3 & \pm 0.2 & a & 0.7 & \pm 0.2 & $\mathrm{~b}$ & $<0.01^{+\dagger}$ \\
\hline Dairy products & 162.6 & \pm 18.7 & a & 194.4 & \pm 5.6 & a & 78.2 & \pm 10.1 & $\mathrm{~b}$ & $<0.001^{t+}$ \\
\hline Oils & 5.8 & \pm 0.8 & $\mathrm{~b}$ & 8.7 & \pm 0.2 & b & 10.7 & \pm 1.3 & $\mathrm{a}$ & $<0.01^{t+}$ \\
\hline Soda & 55.1 & \pm 16.4 & $\mathrm{~b}$ & 46.8 & \pm 2.7 & $\mathrm{~b}$ & 614.7 & \pm 17.8 & $\mathrm{a}$ & $<0.001^{t+}$ \\
\hline SSBs & 69.8 & \pm 21.8 & & 81.7 & \pm 4.5 & & 78.6 & \pm 15.7 & & \\
\hline Processed foods & 29.9 & \pm 3.7 & & 31.9 & \pm 0.8 & & 32.8 & \pm 2.5 & & \\
\hline
\end{tabular}


Values are presented as Mean \pm SE

${ }^{+}$P-values for differences using ANCOVA and adjusting for age, sex, and total energy intake (kcal).

${ }^{\dagger+}$ Different superscript letters represent the results of the post hoc tests. Adjustment for multiple comparisons: Bonferroni

More specifically, higher total energy, protein, and fat consumption rates were shown in the western-style fast-food group. Among the food groups, higher consumption rates of white rice, wheat/bread, eggs, fish, and dairy products are shown in the ready-to-eat group $(\mathrm{p}<0.05)$ whereas, in the balanced group, higher consumption rates of whole grains, vegetables, fruits, seaweed, and dairy products were found $(p<0.05)$. By contrast, a higher intake of wheat/bread, meat, meat products, oils, and soda was reported in the western-style fast-food $(\mathrm{p}<0.05)$.

A multivariable adjusted regression analysis was performed to evaluate the associations between dietary patterns and HGS. The results are presented as adjusted coefficients and $95 \%$ confidence intervals for the ready-to-eat and westernstyle fast-food patterns, which were compared with the balanced pattern (Table 3). 
Table 3

Differences in food intake of 10 to 18-year-old children and adolescents according to the three dietary patterns.

\begin{tabular}{|c|c|c|c|c|c|c|c|c|}
\hline & \multicolumn{3}{|c|}{ ready-to-eat } & \multirow[t]{3}{*}{$p$} & \multirow{2}{*}{\multicolumn{3}{|c|}{$\begin{array}{l}\text { western-style fast-food } \\
217(9.3)\end{array}$}} & \multirow[t]{3}{*}{$p$} \\
\hline \multirow[t]{2}{*}{$\mathbf{N}(\%)$} & \multicolumn{3}{|c|}{1992 (85.6) } & & & & & \\
\hline & $B$ & SE & $\mathrm{Cl}$ & & $B$ & SE & $\mathrm{Cl}$ & \\
\hline \multicolumn{9}{|c|}{ Food groups(g/day) } \\
\hline White rice & 89.0 & 33.9 & $(22.5,155.4)$ & s. 001 & $\overline{118.3}$ & 44.2 & $\begin{array}{l}(-205.0,- \\
31.5)\end{array}$ & <. 001 \\
\hline Wholegrain & -22.1 & 13.9 & $(-49.5,5.2)$ & & -47.9 & 16.5 & $(-80.2,-15.6)$ & $<0.05$ \\
\hline Wheat/bread & 87.7 & 32.6 & $(23.6,151.8)$ & $<0.01$ & 128.4 & 41.0 & $(47.7,209.0)$ & $<0.01$ \\
\hline Noodle & 18.5 & 30.7 & $(-41.7,78.7)$ & & -26.0 & 38.0 & $(-100.6,48.7)$ & \\
\hline Potatoes & -10.0 & 12.0 & $(-33.7,13.6)$ & & 12.6 & 14.5 & $(-15.9,41.0)$ & \\
\hline Sugars & 13.7 & 8.5 & $(-3.0,30.3)$ & & 15.7 & 11.6 & $(-7.0,38.5)$ & \\
\hline Beans & -5.1 & 6.1 & $(-17.1,6.9)$ & & -9.5 & 7.2 & $(-23.7,4.7)$ & \\
\hline Nuts & -6.5 & 4.4 & $(-15.1,2.1)$ & & -5.3 & 5.1 & $(-15.3,4.7)$ & \\
\hline Vegetables & -3.1 & 6.7 & $(-16.3,10.1)$ & & -11.6 & 7.5 & $(-26.4,3.1)$ & \\
\hline Kimchi & 0.7 & 2.0 & $(3.2,4.5)$ & & -1.1 & 2.6 & $(-6.2,4.1)$ & \\
\hline Mushrooms & -1.4 & 1.4 & $(-4.1,1.3)$ & & -2.1 & 1.5 & $(-5.1,0.9)$ & \\
\hline Fruits & $\overline{3} 26.7$ & 15.9 & $\begin{array}{l}(-357.9,- \\
259.5)\end{array}$ & $<.000$ & $\overline{3} 44.4$ & 17.1 & $\begin{array}{l}(-377.9,- \\
310.8)\end{array}$ & <. 000 \\
\hline $\begin{array}{l}\text { Meat and its } \\
\text { products }\end{array}$ & 113.0 & 27.5 & $(59.1,166.9)$ & $\begin{array}{l}< \\
0.001\end{array}$ & 244.2 & 41.3 & $(163.1,325.4)$ & $\dot{0} .001$ \\
\hline Eggs & 0.4 & 8.0 & $(-15.3 .16 .2)$ & & -17.8 & 9.1 & $(-35.7,0.1)$ & \\
\hline Fish & 2.3 & 7.3 & $(-12.0,16.6)$ & & -15.2 & 9.5 & $(-33.8,3.4)$ & \\
\hline Seaweed & 0.1 & 0.9 & $(-1.7,2.0)$ & & -2.3 & 1.0 & $(-4.4,-0.3)$ & $<0.05$ \\
\hline Dairy products & 25.2 & 19.9 & $(-14.0,64.4)$ & & -67.4 & 22.3 & $\begin{array}{l}(-111.3,- \\
23.6)\end{array}$ & $<0.05$ \\
\hline Oils & 25.0 & 7.0 & $(11.1,38.8)$ & <. 001 & 42.8 & 13.3 & $(16.6,69.0)$ & $<0.01$ \\
\hline Soda & -2.2 & 6.9 & $(-15.6,11.3)$ & & 236.1 & 11.0 & $(214.4,257.8)$ & $\begin{array}{l}<.001 \\
0.00\end{array}$ \\
\hline SSBS & 1.3 & 13.9 & $(-26.1,28.7)$ & & -5.3 & 15.6 & $(-36.0,25.4)$ & \\
\hline Processed foods & 3.1 & 8.8 & $(-14.3,20.4)$ & & 11.7 & 10.5 & $(-9.0,32.4)$ & \\
\hline
\end{tabular}

Values are presented as Mean \pm SE.

${ }^{*} \mathrm{p}<0.05,{ }^{*} \mathrm{p}<0.01,{ }^{* \star} \mathrm{p}<0.001$ adjusting variables: age, sex, BMI, energy intake (kcal), METS and house income. Adjustment for multiple comparisons: Bonferroni. 


\begin{tabular}{|c|c|c|c|c|c|c|c|c|}
\hline & \multicolumn{3}{|c|}{ ready-to-eat } & \multirow[t]{3}{*}{$p$} & \multirow{2}{*}{\multicolumn{3}{|c|}{$\begin{array}{l}\text { western-style fast-food } \\
217(9.3)\end{array}$}} & \multirow[t]{3}{*}{$p$} \\
\hline \multirow[t]{2}{*}{$N(\%)$} & \multicolumn{3}{|c|}{$1992(85.6)$} & & & & & \\
\hline & B & SE & $\mathrm{Cl}$ & & $B$ & SE & $\mathrm{Cl}$ & \\
\hline \multicolumn{9}{|l|}{ Nutrients } \\
\hline $\begin{array}{l}\text { Total energy } \\
\text { (kcal/day) }\end{array}$ & $\overline{369.0}$ & 82.1 & $\begin{array}{l}(-530.3,- \\
207.8)\end{array}$ & $\hat{0} .001$ & 201.4 & 106.7 & $(-8.2,411.0)$ & \\
\hline Protein (g/day) & -4.6 & 3.2 & $(-11.0,1.7)$ & & 20.2 & 5.4 & $(9.7,30.8)$ & $\hat{0} .001$ \\
\hline Fat (g/day) & 1.3 & 2.6 & $(-3.8,6.4)$ & & 25.0 & 4.1 & $(17.0,33.1)$ & $\hat{0} .001$ \\
\hline $\begin{array}{l}\text { Carbohydrate } \\
\text { (g/day) }\end{array}$ & $\overline{100.6}$ & 15.4 & $\begin{array}{l}(-130.9,- \\
70.2)\end{array}$ & $\hat{0} .001$ & -40.3 & 18.2 & $(-75.9,-4.6)$ & $<0.05$ \\
\hline Fiber (g/day) & -13.6 & 1.8 & $(-17.2,-10.0)$ & $\hat{0} .001$ & -12.0 & 2.0 & $(-15.9,-8.1)$ & $\hat{0} .001$ \\
\hline Calcium (mg/day) & -70.8 & 31.1 & $(-131.8,-9.7)$ & $<0.05$ & $\overline{118.0}$ & 36.4 & $\begin{array}{l}(-189.5,- \\
46.5)\end{array}$ & $<0.01$ \\
\hline $\begin{array}{l}\text { Vitamin A } \\
\text { (ugRE/day) }\end{array}$ & $\overline{112.1}$ & 111.1 & $(-330.4,106.1)$ & & $\overline{118.6}$ & 122.4 & $\begin{array}{l}(-358.9 \\
121.8)\end{array}$ & \\
\hline Vitamin C (mg/day) & $\overline{145.4}$ & 21.7 & $\begin{array}{l}(-187.9,- \\
102.9)\end{array}$ & $\hat{0} .001$ & $\overline{142.5}$ & 22.5 & $\begin{array}{l}(-186.7,- \\
98.3)\end{array}$ & $\hat{0} .001$ \\
\hline \multicolumn{9}{|c|}{ Values are presented as Mean \pm SE. } \\
\hline $\begin{array}{l}* \mathrm{p}<0.05,{ }^{* *} \mathrm{p}<0.01 \\
\text { Adjustment for } \mathrm{mu}\end{array}$ & 0.00 & us & iables: age, & $\mathrm{Ml}$ & & & & \\
\hline
\end{tabular}

Compared with the balanced dietary pattern, the ready-to-eat and western-style fast-food patterns were shown to have lower nutrient content. Notably, higher intakes of wheat/bread, meat (including its derivatives), and oils, as well as lower intakes of fruit, were shown in the ready-to-eat and western-style fast-food patterns; in addition, these two last patterns were also shown to imply a lower intake of carbohydrates, fibers, calcium, and vitamin $\mathrm{C}$ than the balanced diet pattern.

Multivariate linear regression analysis was used to evaluate the association between dietary patterns and HGS and HGSWR in the 10-18-year-old children and adolescents, as presented in Table 4. Both Model 1 (i.e., adjustment for age, sex, and house income) and Model 2 (i.e., adjustment for age, sex, BMI, energy intake (kcal), METs, and house income) were inversely associated with HGS and HGSWR $(p<0.05)$. Compared with the balanced pattern, the ready-to-eat pattern was shown to be associated with significantly lower HGS and HGSWR. However, no significant association was found between HGS or HGSWR in the western-style fast-food pattern compared to balanced pattern. 
Table 4

Multivariate linear regression analysis of the association between dietary patterns and HGS of 10-18-year-old children and adolescents.

\begin{tabular}{|c|c|c|c|c|c|c|c|c|}
\hline \multirow[t]{3}{*}{$N(\%)$} & \multirow{2}{*}{\multicolumn{3}{|c|}{$\begin{array}{c}\text { ready-to-eat } \\
1992(85.6)\end{array}$}} & \multirow[t]{3}{*}{$p$} & \multicolumn{3}{|c|}{ western-style fast-food } & \multirow[t]{3}{*}{$p$} \\
\hline & & & & & \multicolumn{3}{|c|}{$217(9.3)$} & \\
\hline & $B$ & SE & $\mathrm{Cl}$ & & $B$ & SE & $\mathrm{Cl}$ & \\
\hline \multicolumn{9}{|l|}{ HGS } \\
\hline Model 1 & -1.6 & 0.7 & $(-2.9,-0.3)$ & 0.0148 & -0.5 & 0.8 & $(-2.1,1.1)$ & 0.5213 \\
\hline Model 2 & -1.3 & 0.6 & $(-2.5,-0.0)$ & 0.0423 & -0.9 & 0.7 & $(-2.3,0.6)$ & 0.2431 \\
\hline \multicolumn{9}{|l|}{ HGSWR } \\
\hline Model 1 & -3.0 & 1.0 & $(-5.1,-0.9)$ & 0.0044 & -1.9 & 1.3 & $(-4.4,0.5)$ & 0.1206 \\
\hline Model 2 & -2.2 & 0.9 & $(-3.9,-0.5)$ & 0.0101 & -1.5 & 1.0 & $(-3.5,0.4)$ & 0.1162 \\
\hline \multicolumn{9}{|c|}{ HGS, handgrip strength; HGSWR, handgrip-strength-to-weight ratio. } \\
\hline \multicolumn{9}{|c|}{${ }^{*} \mathrm{p}<0.05, * * \mathrm{p}<0.01, * * * \mathrm{p}<0.001$} \\
\hline \multicolumn{9}{|c|}{ Model 1 = adjustment for age, sex, and house income. } \\
\hline
\end{tabular}

\section{Discussion}

According to our results, there was a higher proportion of more ready-to-eat dietary patterns than we expected in Korean children and adolescents, particularly ready-to-eat dietary patterns with high consumption of white rice, wheat/bread, eggs, fish, and dairy products. The ready-to-eat dietary pattern seems to have unhealthy effects on children and adolescents due to its hypocaloric and nutrient-poor content. Moreover, HGS was lower in participants from the ready-toeat group than those from the balanced and western-style fast-food groups. Further, following adjustment for potential confounders, HGSWR was higher in participants following a balanced or western-style fast-food dietary pattern rather than in those following a ready-to-eat dietary pattern. McNaughton et al. showed that a dietary pattern rich in fruit, salad, cereals, and fish might be associated with diastolic BP in 1,086 adolescents.(27) Moreover, recent studies showed that greater HGS is associated with longitudinal health maintenance and health improvements in adolescents(28); in addition, the author reported that low HGS could serve as a prognostic indicator of cardiometabolic risk and to identify adolescents who would benefit most from lifestyle interventions to improve muscular fitness.(28) Remarkably, our results agree with those of the aforementioned studies. However, in the present study, the additional evaluation of HGSWR deepened the investigation of muscle quality; besides, HGSWR seems to be also associated with dietary intake of adolescents, especially with the ready-to-eat dietary pattern.

Furthermore, the participants following a ready-to-eat or western-style fast-food dietary pattern showed a lower consumption of carbohydrates, fibers, calcium, and vitamin C compared to those following a balanced dietary pattern. The ready-to-eat dietary pattern implies a high intake of eggs, fish, processed products, and refined carbohydrates such as white rice, flour, and bread - as well as a low intake of vegetables and fruits, which is rather common for foods from convenience or street-food stores. By contrast, the balanced dietary pattern is crucial for adolescents, especially when active physical and emotional development is considered. Nevertheless, Korean adolescents seem to prefer simple 
meals such as bread, sandwiches, and cereals, and the frequency of convenient dietary patterns is increasing.(29) In addition, the data from The Korean Student Health Examination and the Korean Youth Risk Behavior Web-based Survey showed that the consumption of healthy foods such as fruits and vegetables tended to decrease while the consumption of foods such as fast food and ramen increased. $(30,31)$ According to the Korean Youth Risk Behavior Web-based Survey $(n=62,276), 39.3 \%$ of the Korean adolescents consume convenient food more than once or twice a week, whereas $26.0 \%$ more than thrice a week. High-school students had a higher intake of convenient food than middleschool students. Adolescents who consumed convenient food more than thrice a week had a lower intake of recommended foods such as fruits, vegetables, and milk as well as higher intakes of fast food, snacks, and soda than the rest of the adolescent population. However, this is not only a problem of Korean adolescents: in fact, the food consumption patterns of Indian adolescents have also shown to imply inadequate dietary intakes, including lower consumption of vegetables and higher consumption of energy-dense snacks.(32) Cutler et al. reported increases in fastfood consumption patterns in older boys and girls.(33) In the United States, fast food is consumed by one-third of children each day and by two-thirds of children every week.(34) In summary, the adolescents' preference for ready-to-eat dietary patterns seems to be a problem worldwide.(35)

The participants following a balanced dietary pattern ate a lot of whole grains, potatoes, beans, nuts, vegetables, mushrooms, fruits, eggs, fish, seaweed, and dairy products, as the name balanced suggests; notably, their HGSWR was significantly higher than that of the participants following either a ready-to-eat dietary pattern. Western-style fast-food dietary patterns, which were based on wheat, bread, noodles, sugars, meats, oils, sodas, and seasonings, were not associated with decreased HGSWR compared to balanced dietary patterns in this study. This may be due to the inclusion of unique Korean eating patterns in the definition of the western-style fast-food pattern group. Remarkably, kimchi - a traditional Korean food - was consumed in all groups; this may deviate from the traditional western-style diet as we know it. In this study, we defined the western-style fast-food dietary pattern as based on wheat/bread, noodles, sugar, meat, oils, sodas, and seasonings. However, other studies defined the western-style diet as based on poultry, eggs, mayonnaise, fast foods, pizza, pies, and fried potatoes. ${ }^{35}$ As a consequence, further studies are still necessary in order to find an association between the western-style diet and HGSWR.

Interestingly, high BMI and low socioeconomic status are known to be associated with decreased muscle strength.(36, 37) However, in this study, a reduction of HGSWR was observed in association with the ready-to-eat group rather than with the balanced group, even with adjustments for BMI and socioeconomic status. Therefore, the results of this study show that dietary patterns could independently play an essential role in muscle quality.

Robinson et al. showed an association between nutrition and sarcopenia and insisted that efforts to prevent muscle strength should be started earlier in life with the optimization of diet and nutrition.(38) Moreover, unhealthy diets are well-known risk factors for several pathologies.(39) Fiber intake, fat, physical activity is introduced as a distal risk factor in chronic disease prevention model, and specific strategies are underway by several countries for the prevention of chronic diseases. Reduction in HGS and HGSWR by participants following a ready-to-eat dietary pattern indicates that Korean adolescents are also experiencing problems due to nutritional imbalance. Further, adolescents need to learn the importance of following a balanced diet and reducing the intake of fast food or ready-to-eat food in early life. In addition, an appropriate environment would be required for the spread of balanced dietary customs. National efforts on nutritional policies are needed for reducing ready-to-eat dietary customs and the overall environment related to nutritional imbalance.

Nonetheless, this study has several limitations. Firstly, errors might occur in the 24-hour dietary recall method in face-toface interviews even when examinations are performed by trained interviewers. Secondly, many common types of food might not be represented in the questionnaire. Thirdly, this is a cross-sectional study, and a longitudinal study might instead be a better method for detecting an association between food intake and HGSWR.

Page $14 / 18$ 
Despite these limitations, this is the first study to investigate the association between HGS, HGSWR, and dietary intake in adolescents.

\section{Conclusions}

In this study, we compared three types of dietary intake patterns with HGS and HGSWR. As a result, we identified an association between HGS and HGSWR and the type of dietary pattern followed by adolescents.

HGS and HGSWR in ready-to-eat dietary patterns were significantly reduced, and it does not just indicate reduced the quality of simple muscles. This implies the possible occurrence of several concurrent problems related to reduced muscle quality, which might be reflected by HGS and HGSWR. Moreover, the overall environmental factors potentially inducing such unhealthy dietary preferences should be investigated, and appropriate lifestyle changes in Korean adolescents should be encouraged.

\section{Abbreviations}

Non-alcoholic fatty liver disease

NAFLD; handgrip strength:HGS; Korean National Health and Nutrition Examination Survey:KNHANES; Korea Center for Disease Control and Prevention:KCDC; metabolic equivalent task:MET; body mass index:BMl; Handgrip-strength-toweight ratio:HGSWR; sugar-sweetened beverages:SSBs; Blood pressure:BP

\section{Declarations}

\section{Ethics approval and consent to participate}

Informed consent was obtained from all participants at the time of the survey. The Kyung Hee Institutional Review Board approved this study protocol (KHSIRB-20-052(EA)).

\section{Consent for publication}

Not applicable

\section{Availability of data and materials}

Data are available at https://knhanes.cdc.go.kr/

\section{Competing interests}

The authors declare no competing interest associated with this report.

\section{Funding}

Not applicable

\section{Authors' contributions}

Koh Hong and Lim Hyunjung were the group leader. Conceptualization was done by Kang Yunkoo, Lim Hyunjung and Koh Hong. Formal analysis performed by Kim Jieun and Do-Yeon Kim. Kim Jieun, Do-Yeon Kim, Kim Seung and Park Sowon worked with software. All authors approved the final manuscript as submitted and agree to be accountable for all aspects of the work. 
Not applicable

\section{References}

1. Chiabi A, Tchokoteu PF, Takou V, Fru F, Tchouine F. Anthropometric measurements of children attending a vaccination clinic in Yaounde, Cameroon. Afr Health Sci. 2008;8(3):174-9.

2. Hardy J, Kuter H, Campbell M, Canoy D. Reliability of anthropometric measurements in children with special needs. Arch Dis Child. 2018;103(8):757-62.

3. Group WHOMGRS. WHO Child Growth Standards based on length/height, weight and age. Acta Paediatr Suppl. 2006;450:76-85.

4. Fry AC, Irwin CC, Nicoll JX, Ferebee DE. Muscular Strength and Power in 3-to 7-Year-Old Children. Pediatr Exerc Sci. 2015;27(3):345-54.

5. Hogrel JY, Decostre V, Alberti C, Canal A, Ollivier G, Josserand E, et al. Stature is an essential predictor of muscle strength in children. BMC Musculoskelet Disord. 2012;13:176.

6. Sandercock GRH, Cohen DD. Temporal trends in muscular fitness of English 10-year-olds 1998-2014: An allometric approach. J Sci Med Sport. 2019;22(2):201-5.

7. Firth J, Firth JA, Stubbs B, Vancampfort D, Schuch FB, Hallgren M, et al. Association Between Muscular Strength and Cognition in People With Major Depression or Bipolar Disorder and Healthy Controls. JAMA Psychiatry. 2018;75(7):740-6.

8. Ervin RB, Fryar CD, Wang CY, Miller IM, Ogden CL. Strength and body weight in US children and adolescents. Pediatrics. 2014;134(3):e782-9.

9. Peterson MD, Saltarelli WA, Visich PS, Gordon PM. Strength capacity and cardiometabolic risk clustering in adolescents. Pediatrics. 2014;133(4):e896-903.

10. Cruz-Jentoft AJ, Bahat G, Bauer J, Boirie Y, Bruyere O, Cederholm T, et al. Sarcopenia: revised European consensus on definition and diagnosis. Age Ageing. 2019;48(1):16-31.

11. Kang Y, Park S, Kim S, Koh H. Handgrip Strength Among Korean Adolescents With Metabolic Syndrome in 20142015. J Clin Densitom. 2018.

12. Ramirez-Velez R, Pena-lbagon JC, Martinez-Torres J, Tordecilla-Sanders A, Correa-Bautista JE, Lobelo F, et al. Handgrip strength cutoff for cardiometabolic risk index among Colombian children and adolescents: The FUPRECOL Study. Sci Rep. 2017;7:42622.

13. Steffl M, Chrudimsky J, Tufano JJ. Using relative handgrip strength to identify children at risk of sarcopenic obesity. PLoS One. 2017;12(5):e0177006.

14. Orsso CE, Tibaes JRB, Oliveira CLP, Rubin DA, Field CJ, Heymsfield SB, et al. Low muscle mass and strength in pediatrics patients: Why should we care? Clin Nutr. 2019;38(5):2002-15.

15. Centers for Disease Control and Prevention. U.S. Obesity Trends. 2011 [Available from: https://www.cdc.gov/obesity/data/databases.html.

16. The U.S. Department of Health \& Human Services.

17. Frazier AL, Li L, Cho E, Willett WC, Colditz GA. Adolescent diet and risk of breast cancer. Cancer Causes Control. 2004;15(1):73-82. 
18. Park MJ, Scott JT, Adams SH, Brindis CD, Irwin CE, Jr. Adolescent and young adult health in the United States in the past decade: little improvement and young adults remain worse off than adolescents. J Adolesc Health. 2014;55(1):3-16.

19. Reavley N, Patton GC, Sawyer SM, Kennedy E, Azzopardi P. Health and Disease in Adolescence. In: rd, Bundy DAP, Silva N, Horton S, Jamison DT, Patton GC, editors. Child and Adolescent Health and Development. Washington (DC)2017.

20. Birch L, Savage JS, Ventura A. Influences on the Development of Children's Eating Behaviours: From Infancy to Adolescence. Can J Diet Pract Res. 2007;68(1):s1-s56.

21. Korea Centers for Disease Control and Prevention. Korea National Health and Nutrition Examination Survey: 20142017. .

22. Oh JY, Yang, Y. J., Kim, B. S., and Kang, J. H. . Validity and Reliability of Korean Version of International Physical Activity Questionnaire (IPAQ) Short Form. Journal of the Korean Academy of Family Medicine. 2007;28(7):532-41.

23. Kim JH, Yun S, Hwang SS, Shim JO, Chae HW, Lee YJ, et al. The 2017 Korean National Growth Charts for children and adolescents: development, improvement, and prospects. Korean J Pediatr. 2018;61(5):135-49.

24. Park S-H, Kim S-N, Lee SH, Choe J-S, Choi Y. Development of 9th Revision Korean Food Composition Table and Its Major Changes. Korean J Community Nutr. 2018;23(4):352-65.

25. Sciences SNAoA. National Academy of Agricultural Sciences. Food Composition Table. 8th revision.2011. 1-636 p.

26. Food Composition Table 9th revision. In: Sciences NloA, editor. 2017.

27. McNaughton SA, Ball K, Mishra GD, Crawford DA. Dietary patterns of adolescents and risk of obesity and hypertension. J Nutr. 2008;138(2):364-70.

28. Ng AK, Hairi NN, Jalaludin MY, Majid HA. Dietary intake, physical activity and muscle strength among adolescents: the Malaysian Health and Adolescents Longitudinal Research Team (MyHeART) study. BMJ Open. 2019;9(6):e026275.

29. Rural Economic Institute [Available from: http://www.krei.re.kr/foodSurvey/selectBbsNttView.do? key $=807 \& b b s N o=450 \& n t t N o=127802 \&$ searchCtgry=\&searchCnd=all\&searchKrwd=\&pagelndex=1\&integrDeptCode= .

30. Korean Educational Development Institute [Available from: http://schoolhealth.kr/web/srs/selectPublicDataList.do? bbsTyCode=pData\&shNum=22\&pageIndex=1\&pageUnit=10\&dataType=\&searchCnd=\&searchWrd $=$.

31. The 13th Korea Youth Risk Behavior Web-based Survey, 2017, Ministry of Education, Ministry of Health and Welfare, Centers for Disease Control and Prevention [Available from: http://yhs.cdc.go.kr.

32. Rathi N, Riddell L, Worsley A. Indian adolescents' perceptions of the home food environment. BMC Public Health. 2018;18(1):169.

33. Cutler GJ, Flood A, Hannan P, Neumark-Sztainer D. Major patterns of dietary intake in adolescents and their stability over time. J Nutr. 2009;139(2):323-8.

34. Kliegman RM. Nelson textbook of pediatrics. 21st edition. ed. Philadelphia, MO: Elsevier; 2019. pages cm p.

35. Boumtje PI, Huang CL, Lee JY, Lin BH. Dietary habits, demographics, and the development of overweight and obesity among children in the United States. Food Policy. 2005;30(2):115-28.

36. Stenholm S, Mehta NK, Elo IT, Heliovaara M, Koskinen S, Aromaa A. Obesity and muscle strength as long-term determinants of all-cause mortality-a 33-year follow-up of the Mini-Finland Health Examination Survey. Int J Obes (Lond). 2014;38(8):1126-32.

37. Shahar S, Vanoh D, Mat Ludin AF, Singh DKA, Hamid TA. Factors associated with poor socioeconomic status among Malaysian older adults: an analysis according to urban and rural settings. BMC Public Health. 2019;19(Suppl 4):549.

Page 17/18 
38. Robinson S, Cooper C, Aihie Sayer A. Nutrition and sarcopenia: a review of the evidence and implications for preventive strategies. J Aging Res. 2012;2012:510801.

39. Cecchini M, Sassi F, Lauer JA, Lee YY, Guajardo-Barron V, Chisholm D. Chronic Diseases: Chronic Diseases and Development 3 Tackling of unhealthy diets, physical inactivity, and obesity: health effects and cost-effectiveness. Lancet. 2010;376(9754):1775-84.

\section{Figures}

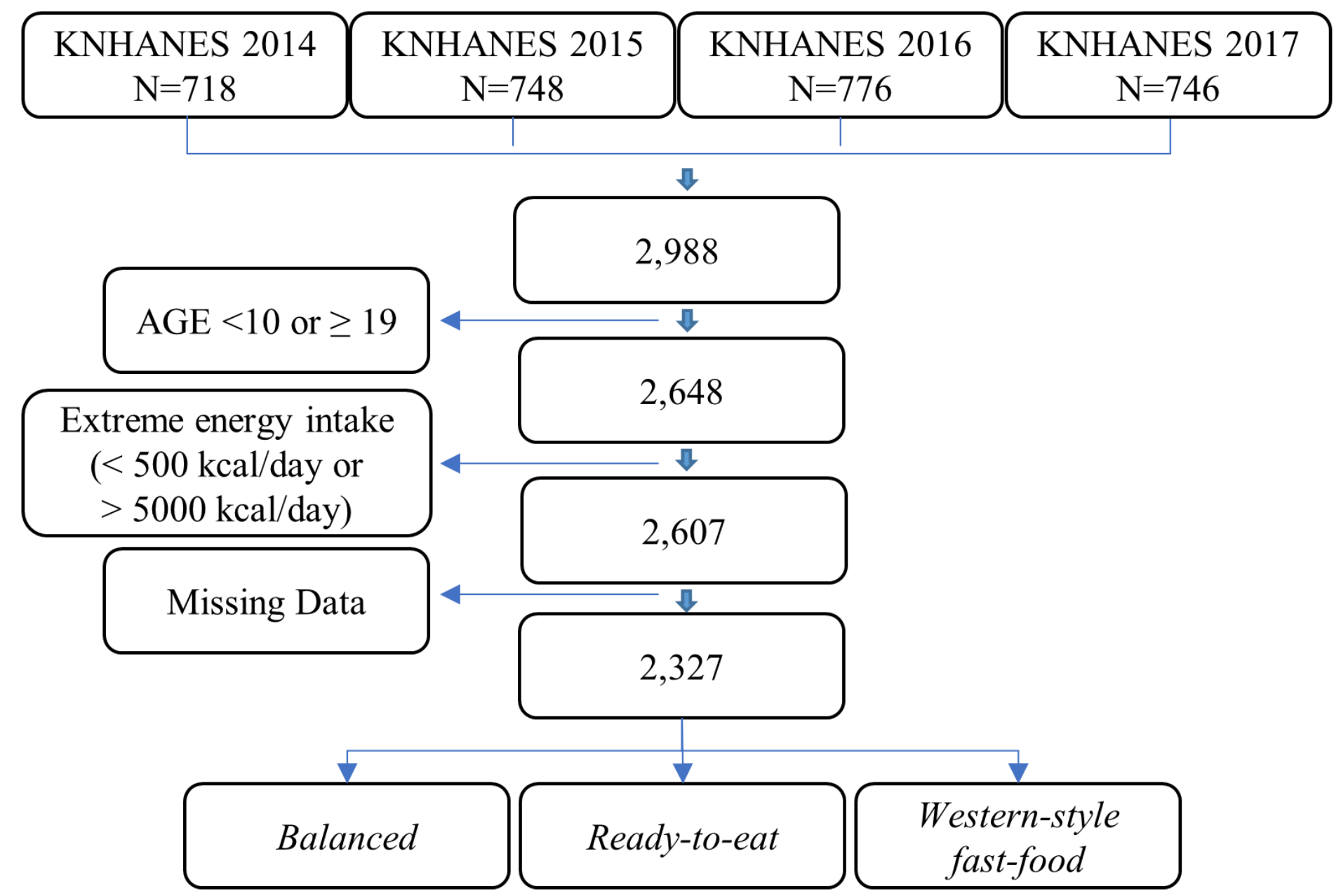

Figure 1

Flow chart for participant selection (2,327 participants). 